\title{
Patrones de apego en la infancia temprana en muestras normativas, contextos de cuidado alternativo, e infancia de alto riesgo
}

\author{
Patterns of attachment in early infancy in normative samples, alternative caregiving \\ systems, and high risk infants
}

\author{
Felipe Lecannelier ${ }^{\mathrm{a}, \mathrm{c}}$, Germán Monje $^{\mathrm{b}, \mathrm{c}}$, Humberto Guajardo ${ }^{\mathrm{a}}$ \\ aFacultad de Ciencias Médicas, Universidad de Santiago de Chile, Santiago de Chile \\ bFacultad de Ciencias Sociales, Universidad Santo Tomás, Puerto Montt, Chile \\ 'Psicólogo
}

Recibido: 8 de enero de 2019; aceptado: 13 de mayo de 2019

\begin{abstract}
Resumen
La evidencia de las últimas décadas ha demostrado que el apego temprano es un proceso vital para la comprensión, prevención e intervención de la salud mental y física de las personas. Sin embargo, es escasa la información sobre el comportamiento del sistema de apego en la realidad chilena. Objetivo: Describir la distribución de los estilos de apego en poblaciones de niños bajo diferentes tipos de cuidado. Sujetos y Método: A través de un método descriptivo retrospectivo, se evaluaron 714 díadas madre-bebé (de 1 a 36 meses) seleccionadas al azar y de modo intencionado, a través de los procedimientos de la Situación Extraña, Escala de Apego durante Stress (ADS), y la Escala de Relación Profesor-Alumno. Las muestras provinieron de familias normativas seleccionadas al azar, infantes de salas de cuna y jardines infantiles JUNJI, e infantes de los centros CONIN y en condiciones de privación de libertad junto a sus madres. Resultados: Las 6 muestras provenientes de 6 estudios demuestran el espectro del comportamiento de los estilos de apego en diversas condiciones: un $70 \%$ seguro y $30 \%$ inseguro, y un $51,1 \%$ seguro y $48.9 \%$ inseguro en muestras normativas; un $48,5 \%$ de seguridad y un $51,5 \%$ de inseguridad en cuidado alternativo y; un 39,6\% de seguridad y $60,4 \%$ inseguridad, y $25 \%$ de estilos seguros y $75 \%$ inseguros en muestras de alto riesgo. Conclusión: El estudio presenta interesantes evidencias sobre las distribuciones de apego en la infancia, que permiten reflexionar sobre la dispar realidad de la situación chilena en lo que a desarrollo socio-afectivo temprano.
\end{abstract}

Palabras clave: Apego; infancia temprana; prevención; riesgo temprano 


\section{Abstract}

Scientific evidence gathered over the last decades has demonstrated that early attachment is a vital process for the understanding, prevention, and intervention of people's mental and physical health. However, information about the attachment system functioning in Chile is scarce. Objective: To describe attachment styles distribution in populations of children under different types of care. Subjects and Method: Through a retrospective descriptive method, 714 mother-child pairs (1 to 36 monthsold) selected at random and purposefully, were assessed using the Strange Situation Procedure, Attachment during Stress Scale (ADS), and Student-Teacher Relationship Scale. The samples were collected from randomly selected normative families, infants attending JUNJI nurseries and kindergartens, and infants from CONIN centers and who live in prison with their mothers. Results: The samples from six studies show the spectrum of the attachment system functioning in diverse conditions: $70 \%$ secure and 30\% insecure, and 51.1\% secure and 48.9\% insecure in normative samples; $48.5 \%$ secure and $51.5 \%$ in secure in alternative care; $39.6 \%$ secure and $60.4 \%$ insecure, and $25 \%$ secure and $75 \%$ in secure styles in high-risk samples. Conclusion: the study presents interesting evidence on the attachment distributions in childhood, which allow reflecting on the uneven Chilean reality with regard to early social and emotional development.

\section{Keywords:}

Attachment; early infancy; preventive interventions; early risk

\section{Introducción}

La Teoría del Apego es considerada como el programa de investigación más completo de las ciencias sociales, tanto por el número de estudios (alrededor de 17.000 investigaciones publicadas), por su alcance a través de todo el ciclo vital (desde el periodo del embarazo hasta la adultez), y la multiplicidad de niveles de análisis (desde lo genético a lo cultural) ${ }^{1,2}$. Sin embargo, son muchas las interrogantes que giran en torno al comportamiento del sistema de apego en diversos contextos y culturas. Una línea importante de investigación busca comparar las distribuciones de los cuatro patrones de apego (Seguro, Inseguro Evitante, Inseguro Ambivalente, Desorganizado) en diferentes culturas y sociedades ${ }^{3}$. En términos generales, se ha evidenciado una tendencia a encontrar $2 / 3$ de patrones seguros, y el tercio restante distribuido en patrones evitantes (12$15 \%)$, ambivalentes (15\%) y desorganizado/desorientado (9\%). Sin embargo, las diferencias culturales son evidentes $^{4}$ : por ejemplo, se han encontrado discrepancias entre los países de Europa del Oeste (hacia la evitación) con relación a Japón e Israel (hacia la ambivalencia $)^{4}$. La conclusión actual es que si bien el sistema de apego tiene una raíz evolutiva-biológica (universal a la especie), su influencia cultural no puede ser olvidada ${ }^{5}$.

Otra línea afín de estudios busca comprender el comportamiento del sistema de apego en condiciones de cuidado alternativo (por ejemplo, salas de cuna) y/o ambientes de alta vulnerabilidad (por ejemplo, institucionalización temprana). En el primer caso, una amplia evidencia ha demostrado que el cuidado alternativo puede tener efectos negativos en el desarrollo del apego (tanto con los cuidadores alternativos como las figuras principales de apego), especialmente cuando el tiempo de estadía en las instituciones supera las 4 horas diarias y la razón de niño:cuidadora es mayor a $6^{6}$. En el segundo caso, estudios en niños maltratados ${ }^{7}$ e institucionalizados tempranamente ${ }^{8}$ ha demostrado que la proporción de $2 / 3$ de apegos seguros y $1 / 3$ de apegos inseguros y desorganizados se invierte, mostrando que este tipo de condiciones es un factor de riesgo extremo para el desarrollo esperable del niño.

No obstante, todos estos estudios se han realizado en países del hemisferio norte, no existiendo evidencia completa sobre la distribución de patrones de apego en muestras normativas y de alto riesgo, y en contextos de cuidado alternativo, en países Latinoamericanos. Por lo anterior, el objetivo del presente estudio es analizar las diferencias en los estilos de apego en diferentes contextos de cuidado. En ese sentido, los resultados presentados permiten representar una panorámica completa del comportamiento del sistema de apego en infantes chilenos, constituyéndose en un aporte único en la región.

\section{Sujetos y Método}

Estudio descriptivo retrospectivo. Para fines de clarificación, los seis estudios se categorizarán de acuerdo a: 1) Población normativa en contexto familiar (estudio 1 y 2);2) Población normativa en cuidado alternativo (salas de cuna y jardines infantiles) (estudio 3 y 4 ); 3) Población de extremo riesgo (institucionalización temprana y madres-bebés privados de libertad) (estudio 5 y 6$)$.

\section{Instrumentos}

Pauta de indicadores sociodemográficos: Se elaboró una pauta de información general que incluyó información relacionada al nombre, edad, sexo, fecha de nacimiento, $\mathrm{y}$ antecedentes médicos generales. 
Procedimiento de la Situación Extraña (PSE) 9 : Procedimiento experimental que consiste en la observación detallada de la interacción de la madre y su infante, a través de 8 episodios de 3 minutos, en los cuáles la madre se separa y se reúne con su hijos, junto a la presencia de una persona extraña ${ }^{11}$. El instrumento se corrige sobre la base de una observación micro-analítica de la conducta de apego del infante, en relación a 4 escalas que se puntúan de 1 a 7 puntos. Las escalas son: 1) búsqueda de proximidad; 2) mantenimiento del contacto; 3) resistencia y frustración; 4) evitación del vínculo. La codificación exhaustiva y entrenada arroja los cuatro patrones de apego: Apego Seguro; Apego Inseguro Evitante; Apego Inseguro Ambivalente; Apego Desorganizado. La codificación del procedimiento fue realizada por el primer autor (FL) quien posee la certificación internacional oficial para dicho propósito (Institute of Child Development, Universidad de Minnesota).

Este procedimiento ha sido considerado el Gold Estándar de los sistemas de evaluación del apego temprano (por ejemplo, 341 estudios publicados hasta el $2010^{10}$, debido a su validez concurrente con otras medidas del desarrollo, predictiva (hasta la edad adulta), y validez multicultural ${ }^{11}$.

Escala de Apego durante Stress de Massie-Campbell (o Escala ADS): Este instrumento es una versión modificada, adaptada y validada de una pauta de observación de indicadores de la calidad del apego en contexto de atención pediátrica ${ }^{12}$, observados tanto en el cuidador principal como en el infante ${ }^{13}$. La pauta de observación incluye 6 indicadores comportamentales de apego (mirada, vocalización, contacto A, contacto B, sosteniendo, afecto y proximidad) que se organizan en una escala de 1 a 5 puntos donde cada puntaje refleja una descripción específica del tipo de comportamiento a utilizar (por ejemplo, un puntaje de 1 en el indicador de "mirada" de la madre representa que "siempre evita mirar a la cara del bebé"). Aquellos puntajes cercanos al 1-2 son indicadores de un apego inseguro evitante, y los puntajes cercanos al 5 son indicadores de apego inseguro ambivalente. Los puntajes cercanos al 3-4 son indicadores de un apego seguro. Diversos estudios arrojaron niveles de validez y confiabilidad adecuados tanto a nivel nacional ${ }^{14}$ como internacional ${ }^{15} \mathrm{y}$ fue utilizada como medida estándar de evaluación del apego (a los 4 y 12 meses de edad de los bebés) en el Programa Nacional de Protección a la Infancia "Chile Crece Contigo", así como en diversos estudios en población de alto riesgo y de eficacia en modelos de intervención temprana ${ }^{16}$. Todas las interacciones fueron grabadas en video y su codificación fue realizada por 2 expertos con más de 5 años de experiencia en el uso de la escala y la observación del apego.
Escala de Relación Profesor-Alumno (StudentTeacher Relationship Scale (STRS $)^{17}$ : El Student Teacher Relationship Scale versión abreviada (STRS-SF), fue validada en Chile por Becar, Cheyre y Diemer ${ }^{18}$, arrojando buenos resultados de confiabilidad y validez factorial. Es una escala Likert, orientada a alumnos(as) entre 4 a 8 años de edad (pero que puede ser utilizada para niños menores de 2 y 3 años), que tiene como propósito evaluar las percepciones que los profesores tienen sobre su relación con un alumno en particular. Por ende evalúa la variable relación emocional entre profesor-alumno(a). Consiste en un cuestionario de autoreporte que responde el profesor. Lo conforman 2 sub-escalas: Conflicto: que mide el grado en que éste percibe su relación con un estudiante como negativa y conflictiva; y, Cercanía: que mide el grado de afecto, calidez y comunicación abierta con un alumno; y una Escala Total que evalúa en general la calidad de la relación.

\section{Procedimiento}

1) Población normativa en contexto familiar (Procedimiento de la Situación Extraña): En los dos estudios ( 1 y 2$)$ se seleccionaron madres-bebés (12 a 20 meses). En cuanto al estudio 1, el proceso de selección se realizó a través de consultorios de atención primaria (Lo Bernechea) y hospitales (Hospital Barros Luco), mientras que en el estudio 2, la selección se llevó a cabo a través de redes sociales y contactos personales. Los criterios de exclusión empleados hicieron alusión a la presencia de psicopatología psiquiátrica grave de las madres, así como también, de enfermedades congénitas del infante que no permitieran la correcta realización de los procedimientos experimentales. Así mismo, se excluyeron madres migrantes. Las madres junto a sus hijos fueron invitados a las dependencias del Servicio de Psicología Integral de la Universidad del Desarrollo y la Unidad de Psiquiatría Sur de la Universidad de Chile, donde previo a una serie de instrucciones y firmas de consentimiento informado se les realizaron las evaluaciones correspondientes (doble espejo), las cuales fueron videograbadas para su posterior análisis, el cual, se realizó en un periodo de 2 meses, para luego finalmente analizar los datos a través del programa estadístico SPSS Versión 19.

Los estudios fueron aprobados por el comité de ética de la Escuela de Psicología de la Universidad del Desarrollo, y financiados por la Comisión de Nacional de Investigación Científica y Tecnológica (CONICYT).

2) Población normativa en cuidado alternativo (salas de cuna y jardines infantiles) (estudio 3 y 4): Ambas muestras fueron seleccionadas dentro de las instituciones de la Junta Nacional de Jardines Infantiles (JUNJI) pertenecientes a las regiones IV, VI, VIII y Metropo- 
litana, como parte de un estudio para evaluar la efectividad de un Programa de Fomento del Apego y el Aprendizaje Socioemocional, financiado por el Ministerio de Desarrollo Social. La edad de los bebés fluctuó entre 1-26 meses (salas de cuna) y 12-53 meses (jardines infantiles). La selección de ambas muestras fue intencionada por las autoridades de JUNJI, por lo que no hubo criterios de exclusión e inclusión. Las videograbaciones se realizaron en el contexto de las salas de cuna y jardines, y la codificación de los videos fue realizada por evaluadores expertos entrenados. El estudio fue aprobado por el comité de ética de la Universidad del Desarrollo y de la JUNJI.

3) Población de extremo riesgo (institucionalización temprana y madres-bebés privados de libertad) (estudios 5 y 6): La primera muestra fue seleccionada desde los centros de atención de Corporación para la Nutrición Infantil (CONIN) que alberga a infantes de 0 a 4 años con diversas patologías crónicas y/o vulnerabilidad psicosocial, con un rango de edad desde los 0 a los 33 meses. Se evaluaron bebés provenientes de centros de 6 ciudades (Arica, Valparaíso, Temuco, Valdivia, Santiago, Los Angeles), y las videograbaciones se realizaron en los centros y la codificación de los videos fue realizada por evaluadores expertos entrenados. La segunda muestra fue seleccionada de 11 centros penitenciarios (Arica, Iquique, Antofagasta, Copiapó, La Serena, Valparaíso, Rancagua, Concepción, Valdivia, Santiago y Los Angeles), y consistió en madres que conviven junto a sus bebés ( 0 a 23 meses). Las diadas fueron evaluadas en los centros penitenciarios, y las videograbaciones se realizaron en los centros; la codificación de los videos fue realizada por evaluadores expertos entrenados. Ambos estudios fueron aprobados por el comité de ética de la Universidad del Desarrollo y de Gendarmería de Chile (GENCHI), y financiado por el Ministerio de Desarrollo Social (Gobierno de Chile), como parte de una investigación para evaluar la efectividad de un Programa para el Fomento del Apego y el Cuidado Respetuoso.

\section{Participantes}

La tabla 1 resume el tamaño de la muestra, la edad (promedio y desviación estándar) y genero de los infantes de los 6 estudios.

\section{Resultados}

La presentación de los resultados se dividirá en dos partes: La descripción de las distribuciones de los estilos de apego en los seis estudios/muestras, y el análisis de la correlación del apego con la edad y género.

En relación con los dos estudios con muestras normativas, se encontró una proporción de $70 \%$ de ape- go seguro y $30 \%$ de apego inseguro y desorganizado (ver tabla 2-estudio 1). En el estudio 2 (tabla 3 ) se encontró un 51,5\% de apego de tipo seguro, y un 48,5\% de apegos inseguros y desorganizados. Así mismo, la proporción de apegos evitantes aumentó desde 10\% a $30 \%$. La proporción de ambivalentes y desorganizados se mantuvo estable.

En lo que respecta a los dos estudios en muestras de cuidado alternativo (estudio 3 y 4), se encontró un $49 \%$ de seguridad en el apego y un $51 \%$ de inseguridad y desorganización (tabla 4). Resalta la alta proporción de apegos de tipo evitante, así como el bajo porcentaje del patrón ambivalente.

Tabla 1. Edad y Desviación Estándar de los niños (en meses) y Tamaño de Muestra

\begin{tabular}{llll}
\hline Estudios & $\overline{\mathrm{x}}$ & \multicolumn{1}{c}{ DS } & $\mathrm{N}$ \\
\hline Estudio 1 (Muestra normativa) & 13,3 & 13,3 & 130 \\
Estudio 2 (Muestra normativa) & 18 & 2,7 & 103 \\
Estudio 3 (Salas cuna) & 17,21 & 5,52 & 156 \\
Estudio 4 (Jardines infantiles) & 36,82 & 7,6 & 288 \\
Estudio 5 (CONIN) & 12,5 & 7,6 & 56 \\
Estudio 6 (GENCHI) & 7,51 & 5,6 & 41 \\
\hline
\end{tabular}

Tabla 2. Distribución de la Clasificación del Apego en el Estudio 1

\begin{tabular}{lc}
\hline Clasificación del Apego & Estudio 1 \\
\hline Seguro & $70 \%$ \\
Evitante & $10 \%$ \\
Ambivalente & $10,8 \%$ \\
Desorganizado & $9,2 \%$ \\
\hline
\end{tabular}

Tabla 3. Distribución de la Clasificación del Apego en el Estudio 2

\begin{tabular}{lc}
\hline Clasificación del Apego & Estudio 2 \\
\hline Seguro & $51,1 \%$ \\
Evitante & $30,9 \%$ \\
Ambivalente & $10,6 \%$ \\
Desorganizado & $7,4 \%$ \\
\hline
\end{tabular}

Tabla 4. Distribución de la Clasificación del Apego en el Estudio 3

\begin{tabular}{lc}
\hline Clasificación del Apego & Estudio 3 \\
\hline Seguro & $49 \%$ \\
Evitante & $36,5 \%$ \\
Ambivalente & $2,1 \%$ \\
Riesgo & $12,5 \%$ \\
\hline
\end{tabular}


En el estudio realizado en el contexto de jardines infantiles, donde se utilizó una metodología diferente a las anteriores, se encontró que las educadoras perciben alto conflicto con el $35 \%$ de sus alumnos, y un $56 \%$ de baja cercanía con ellos (tabla 5).

Finalmente, los estudios en muestras de alto riesgo (estudio 5 y 6 , ver tablas 6 y 7 ), se encontró una inversión del patrón normativo de distribución del apego ( $2 / 3$ de seguridad y $1 / 3$ de inseguridad y desorganización). En el caso de la muestra de los centros CONIN de bebés institucionalizados se encontró un $24,5 \%$ de riesgo (tendencia a la desorganización), y un 26,4\% de estilo evitante (la proporción total de inseguros fue de $60,3 \%)$. En el caso de la muestra de bebés privados de libertad junto a sus madres se encontró un $52,5 \%$ de apegos de alto riesgo y solo un $25 \%$ de apego seguro. Ambos resultados evidencian un desarrollo altamente grave en infantes que todavía no llegan a su segundo año de vida, por lo que pone una voz de alerta en ambas condiciones de alta vulnerabilidad.

El objetivo del segundo tipo de análisis fue confirmar la hipótesis de que los patrones de apego no se relacionan a la edad y el género del bebé (20). A través de una serie de correlaciones no se encontraron en ninguno de los estudios una relación significativa entre el apego y este tipo de variables.

\section{Discusión}

Los resultados arrojados en los seis estudios muestran interesantes discusiones y conclusiones para la dispar realidad de la infancia en Chile (y posiblemente en Latinoamérica). En relación a las dos muestras normativas, cabe discutir dos aspectos claves: En primer lugar, se confirmó la tendencia occidentalizada de los $2 / 3$ de apego seguro y $1 / 3$ de apego inseguro y desorganizado ${ }^{4}$. La discusión actual de si este patrón distributivo es algo más bien universal o propio de cada cultura $^{3,5}$ plantea la necesaria reflexión sobre si Chile se estaría enmarcando más bien dentro de las sociedades del tipo individualista-occidentales, o de si estas dos distribuciones encontradas siguen obedeciendo a criterios más bien biológico-universalistas ${ }^{21}$. En ese sentido, una serie de estudios multiculturales realizados en 24 países sobre temperamento, 16 en salud mental y 16 en crianza, confirmarían la primera hipótesis ${ }^{22-24}$, por lo que Chile estaría alcanzando un patrón de dificultades de adaptación propio de las sociedades industrializadas individualistas. En segundo lugar, y relacionado a lo anterior, es notorio el aumento de los estilos de apego evitantes en el curso de 10 años. Nuevamente, cabe la discusión con respecto a si esta distribución obedecería a esta tendencia hacia el individualismo que al parecer experimenta nuestro país. Por ejemplo, se ha visto que
Tabla 5. Nivel de Cercanía y Conflicto en el Estudio 4

\begin{tabular}{cccc}
\hline \multicolumn{2}{c}{ Nivel de Conflicto } & \multicolumn{2}{c}{ Nivel de Cercanía } \\
\hline Alto & $35 \%$ & Alto & $0 \%$ \\
Normal & $50 \%$ & Normal & $44 \%$ \\
Bajo & $15 \%$ & Bajo & $56 \%$ \\
\hline
\end{tabular}

\section{Tabla 6. Distribución de la Clasificación del Apego} en el Estudio 5

\begin{tabular}{lc}
\hline Clasificación del Apego & Estudio 5 \\
\hline Seguro & $39,6 \%$ \\
Evitante & $26,4 \%$ \\
Ambivalente & $9,4 \%$ \\
Riesgo & $24,5 \%$ \\
\hline
\end{tabular}

Tabla 7. Distribución de la Clasificación del Apego en el Estudio 6

\begin{tabular}{lc}
\hline Clasificación del Apego & Estudio 6 \\
\hline Seguro & $25 \%$ \\
Evitante & $15 \%$ \\
Ambivalente & $7,5 \%$ \\
Riesgo & $52,5 \%$ \\
\hline
\end{tabular}

los estilos evitantes suelen estar asociados a dificultades en salud mental de tipo internalizante (depresión, ansiedad, timidez, etc.), así como también, relacionados con un sobrecontrol emocional, y los resultados multiculturales en lo que ha participado nuestro país han arrojado una prevalencia del 16-20\% de trastornos internalizantes en preescolares chilenos, lo cual supera en hasta tres veces la prevalencia mundial ${ }^{21}$.

Igualmente, los estudios en salas de cuna y jardines infantiles chilenos, siguen confirmando esta tendencia Chilena hacia la evitación/internalización. Los resultados en salas de cuna resultan especialmente preocupantes al encontrar una proporción de $37,1 \%$ de apegos evitantes (lo que sumado al 12,4\% de apegos de riesgo, hacen un $47,5 \%$ de apegos inseguros ya antes de los 12 meses). Durante el primer año, la teoría indica que el sistema de apego se encuentra en su etapa de formación, y nuestra tasa de apegos evitantes (y de bajos apegos ambivalentes) lleva a concluir que, o los bebés ingresan a las salas de cuna con una tendencia hacia la evitación, o la desarrollan en dichos lugares como producto del tipo de cuidado ejercido, o ambas. Tras un análisis realizado sobre el tipo de cuidado de las educadoras bajo una escala de micro-observación, se confirmó la segunda hipótesis, es decir, el trato de 
las cuidadoras está más bien centrado en las necesidades básicas del bebé, pero en mucha menor medida en sus necesidades socioemocionales. En los contextos educativos de jardines infantiles, se encontró que las educadoras reportan un alto conflicto con el 35\% de sus alumnos, y una baja cercanía con el $44 \%$. Ambos datos también resultan preocupantes considerando que la evidencia ha demostrado que altos niveles de conflicto predicen un clima educativo negativo y bajo rendimiento académico ${ }^{25}$. Al respecto cabría analizar $\mathrm{si}$ el estilo de enseñanza y expectativas de las educadoras presiona a un tipo relación conflictiva cuando los infantes expresan su estrés. Lo anterior ha sido confirmado en dos hallazgos donde entrevistando a educadoras se encontró que un alto porcentaje alberga expectativas de que un niño adecuado es "controlado, bien comportado, no llora, no molesta y se queda tranquilo" ${ }^{\text {"2 }}$. Así mismo, en el presente estudio se encontró que alrededor del 40-60\% de las educadoras posee creencias que son opuestas a lo que se podría llamar "crianza respetuosa"27. Cabe remarcar que ambos estudios confirman la hipótesis de un patrón evitante/internalizante de la sociedad chilena.

Finalmente, las muestras de extremo riesgo vienen a confirmar los devastadores efectos de ambientes adversos en el desarrollo del apego temprano. El estudio de los niños de CONIN (institucionalización temprana) confirman los resultados internacionales donde solo $1 / 3$ presenta apego seguro, y $2 / 3$ inseguro o desorganizado ${ }^{8}$. La diferencia y aporte fundamental de esta investigación, es que a diferencia de la gran mayoría, los resultados se obtuvieron antes del primer año y con los educadores de trato directo (ETD). Ambos aspectos resaltan el doble hecho de que mientras más temprana es la institucionalización, más riesgo se produce, y que, a menos que las ETD se especialicen en un cuidado sensible, no se pueden constituir en un ambiente esperable mínimo para el desarrollo adecuado del infante ${ }^{8}$. El estudio nacional de madres y sus bebés privadas de libertad viene a realizar un importante aporte a la línea de investigación en apego y cárcel. Por un lado, el nivel de apego de riesgo encontrado 52,5\%, no solo es altamente preocupante, sino que se constituye en una de las proporciones más altas encontradas en estudios de alto riesgo. Lo anterior obliga a pensar sobre las condiciones (emocionales más que físicas) de cuidado de las madres. Por el otro, se ha demostrado que la permanencia del bebé con sus madres es un factor protector contra re-incidencia en el delito ${ }^{28}$, y que una intervención adecuada sobre el cuidado respetuoso puede aminorar los efectos de riesgo en el apego y el desarrollo socioemocional de los niños ${ }^{28}$. Por ende, estos resultados muestran, por un lado, los efectos altamente perjudiciales del contexto de privación de libertad, y por el otro, la urgencia de la implementación de programas de intervención que sean continuos, ordenados y efectivos en estos contextos.

\section{Responsabilidades Éticas}

Protección de personas y animales: Los autores declaran que los procedimientos seguidos se conformaron a las normas éticas del comité de experimentación humana responsable y de acuerdo con la Asociación Médica Mundial y la Declaración de Helsinki.

Confidencialidad de los datos: Los autores declaran que han seguido los protocolos de su centro de trabajo sobre la publicación de datos de pacientes.

Derecho a la privacidad y consentimiento informado: Los autores han obtenido el consentimiento informado de los pacientes y/o sujetos referidos en el artículo. Este documento obra en poder del autor de correspondencia.

\section{Conflicto de intereses}

Los autores declaran no tener conflicto de interés.

\section{Financiamiento}

Comisión de Nacional de Investigación Científica y Tecnológica (CONICYT), Proyecto Fondecyt $N^{\circ}$ 1140265 y Ministerio del Desarrollo Social.

\section{Referencias}

1. Cassidy J, Shaver, PhR. (ed). Handbook of attachment, Third Edition: Theory, Research, and clinical applications. $3^{\text {nd }}$ Edition. New York: The Guilford Press, 2018.

2. Lecannelier F. Apego e Intersubjetividad: El legado de los vínculos tempranos en el desarrollo humano y la salud mental.
Segunda Parte. La teoría del Apego. Santiago de Chile, Editorial LOM, 2009.

3. Mesman, J, van Ijzendoorn, M, SagiSchwartz, A. Cross-cultural pattern of attachment: Universal and contextual dimensions. En Cassidy J, Shaver, PhR, editors, Handbook of attachment, Second Edition: Theory, Research, and clinical applications. $3^{\text {nd }}$ Edition. New York: The Guilford Press; 2018, p. 827-55.
4. van Ijzendoorn, Zagi-Schwartz, A. Crosscultural patterns of attachment. Universal and contextual dimensions. En Cassidy J, Shaver PhR, editores, Handbook of Attachment. Theory, Research and Clinical Applications. New York: Guilford Press; 2008, p. 880-905.

5. Lecannelier F. La Teoría del Apego: Una mirada actualizada y la propuesta de nuevos caminos de exploración. 
Aperturas Psicoanaliticas, 2018; 58, rescatado en http://aperturas.org/articulo. php?articulo $=0001026 \#$ contenido.

6. Belsky J. Early child care and early child development: Majors findings of the NICHD Study of early child care. Eur J Dev Psych. 2006; 3: 35-78.

7. van Ijzendoorn $\mathrm{MH}$, Schuengel C, Bakermans-Kranenburg M. Disorganized attachment in early childhood: Metaanalysis of precursors, concomitants, and sequelae. Dev Psychopathol. 2009;11:22550.

8. Bakermans-Kranenburg MJ, Steele H, Zeanah Ch, Muhamedrahimov RJ, Vorria P, Dobrova-Krol NA, Steele M, van Ijzendoorn M.H, Juffer F, Gunnar MR. Attachment and emotional development in institutional care: Characteristics and catch-up. Monogr. Soc Res Child Dev. 2011;76:62-91.

9. Ainsworth MDS, Blehar MC, Waters E, Wall S. Patterns of Attachment: A Psychological Study of the Strange Situation. Hillsdale, NJ: Erlbaum, 1978.

10. Simonelli A, De Palo F, Parolin M, Moretti M. Interactive behaviors and attachment patterns in the strange situation procedure: A validation of the Ainsworth model. Psychol Beh Sci. 2014;3:75-84.

11. Solomon J, George C. The measurement of attachment security and related constructs in infancy and early childhood. En Cassidy J, Shaver, PhR, ditors, Handbook of attachment, Second Edition: Theory, Research, and clinical applications. $3^{\text {nd }}$ Edition. New York: The Guilford Press; 2018, p. 366-98.

12. Massie HN and Campbell BK. The Massie-Campbell scale of mother-infant attachment indicators during stress In Call J, Galenson E, and Tyson R editors. Frontiers of infant psychiatry. New York: Basic Books;1983. pp 394-412.

13. Lecannelier F, Zamora C. Escala de Apego durante Stress (ADS-III). Tercera versión:
Manual de administración, corrección y guía de conductas. Santiago: Universidad del Desarrollo, 2013.

14. Acuña E, Maturana P, Muñoz A. Adaptación y validación de la Escala de Apego durante Estrés (ADS) en díadas madre-bebé chilenas. Tesis para optar al grado de magister en Psicologia clínica. Facultad de Psicología, Universidad del Desarrollo, 2011.

15. Carcamo R, van Ijzendoorn $M$, Vermeer $H J$, van der Veer R. The validity of the Massie-Campbell Attachment during Stress Scale (ADS). J Child Fam Stud, DOI 10.1007/s10826-013-9728-z.

16. Lecannelier F, Silva J, Morales R, Melo R, Hoffmann M. Effects of an intervention to promote socioemotional development in terms of attachment security: a study in early institutionalization in Chile. Infant Ment Health J. 2014;35:151-9.

17. Pianta RC. STRS, Student-Teacher Relationship Scale, Professional Manual. Lutz: Psychological Assessment Resources, 2001.

18. Becar C, Cheyre A, Diemer M. Adaptación y validación de una escala para evaluar la relación afectiva entre profesores y alumnos entre 4 y 8 años de edad: Escala de Relación Afectiva Profesor-Alumno (Student-Teacher Relationship Scale Short Form, STRS-SF). Tesis para optar al grado de magister en Estrategias en Salud Mental Infanti. Facultad de Psicología, Universidad del Desarrollo, 2011.

19. Lecannelier F, Kimelman M, González L, Núñez C. Hoffmann M. Evaluación de patrones de apego en infantes durante su segundo año en dos centros de atención de Santiago de Chile. Rev Argent Clín Psicol. 2008; XVII: 197-207.

20. De Wolff MS, van Ijzendoorn MH. Sensitivity and attachment: A metaanalysis on parental antecedents of infant attachment. Child Dev. 1997;68:571-91.
21. Krassner AM, Gartstein MA, Park C, Dragan W, Lecannelier F, Putnam S. East-West collectivist-individualist: A cross-cultural examination of temperament in toddlers from Chile, Poland South Korea, and the US. Eur J Dev Psych. 2017;14:449-64.

22. Rescorla N, Achenbach TM, Ivanova MY, et al. International comparisons of behavioral and emotional problems in preschool children: Parent's report from 24 societies. J Clin Child Adolesc Psychol. 2012; 40:456-67.

23. Rescorla N, Achenbach TM, Ivanova MY, et al. Behavioral/emotional problems of preschoolers: Caregiver/teacher reports from 15 societies. J Emot Behab Disord. 2012; 20: 68-81.

24. Gartstein M, Putnam S. Toddlers, parents, and culture. Findings from the Joint Effort Toddler Temperament Consortium. Routledge, 2018.

25. Pianta RC. Enhancing relationship between children and teachers. Washington, DC: American Psychological Association, 1999.

26. Garrido AM, Weitzel CP. Significaciones en educadoras y auxilizares de párvulo respecto del aprendizaje socioafectivo en la educación parvularia. Tesis para optar al grado de magister en Estrategias en Salud Mental Infanti. Facultad de Psicología, Universidad del Desarrollo, 2011.

27. Lecannelier F, Jorquera L, Silva J. Diseño metodológico para el fortalecimiento del apego seguro, desarrollo socioemocional, y crianza respetuosa en la intervención de niños y niñas salas de cuna y jardines infantiles: Los Modelos AM.ARCuidadores y A.M.A.R.-Educadores. Ministerio de Desarrollo Social, Gobierno de Chile, 2014.

28. Murray J, Murray L. Parental incarceration, attachment and psychopathology. Attach Hum Dev. 2010;12:289-309. 